the results of which check Thomas' eigenvalues and eigenfunctions to four significant numbers. The eigenfunctions of the critical points of the neutral stability curves for $A=0.4367$ and $A=0$ are shown in Fig. 5. The inner and outer viscous layers, discussed by Lin, ${ }^{3}$ have approximately the same thicknesses for this extreme case as for the Poiseuille flow which, according to $\mathrm{Lin}$, is reasonable since these thicknesses depend on the parameter $\alpha R$.

\section{ACKNOWLEDGMENTS}

The basic computer program which was used in this study was developed by Dr. W. C. Reynolds of Stanford University, under whose direction the author participated in a National Science Foundation Summer Research Participation for College Teachers Program. The time donated by the Computer Center of Michigan State University was very much appreciated.

\title{
Two-Dimensional Gravity Waves in a Stratified Ocean
}

\author{
T. YAO-TsU WU \\ California Institute of Technology, Pasadena, California \\ and \\ C. C. MEI \\ Massachusetts Institute of Technology, Cambridge, Massachusetis
}

(Received 19 September 1966)

\begin{abstract}
The solution of the two-dimensional gravity waves in a plane stratified ocean previously calculated by the authors is rectified. By formulating a corresponding initial value problem, the steady-state solution becomes completely determinate without using the radiation condition.
\end{abstract}

\section{INTRODUCTION}

$\mathbf{I}^{\mathrm{I}}$ $\mathrm{N}$ a previous paper, ${ }^{1}$ the authors considered the fundamental solution of the two-dimensional gravity waves due to a local disturbance in a stratified ocean. The surface and the internal wave modes were determined for two cases of density stratification, one of them corresponding to constant Vasala frequency (with density increasing exponentially with depth) and the other to a monotonically decreasing Vasala frequency with inareasing depth. The surface wave mode, which propagates downstream without attenuation, was studied in detail there. The conclusion regarding the attenuated internal waves is, however, found in error. Treating the two-dimensional flow problem when the point disturbance travels horizontally at a constant velocity, the authors adopted a steady-state formulation which does not yield a unique solution unless further physical conditions are imposed on the radiation of the generated waves. While the surface wave mode was calculated correctly by using the radiation condition, the internal wave mode was determined

I C. C. Mei and T. Y. Wu, Phys. Fluids 7, 1117 (1964). erroneously. This error is due to an incorrect choice of the path for a Fourier integral, thus leading to the wrong result that the internal waves appear symmetrically on both the upstream and downstream sides, and that they are attenuated in the horizontal direction and hence radiate no energy to infinity. Similar errors have been made in the past for atmospheric waves ${ }^{2}$ and a clarification by the approach of an initial value problem has been given by Crapper. ${ }^{3}$

The present note is intended to rectify the previous incorrect result by obtaining the steady-state solution as the large time limit of a corresponding initial value problem. In this limit of the solution, which is expressed in an integral representation, the path for the contour integral around a simple pole and two branch points can be determined definitely. It is now found that the internal waves in this twodimensional stratified flow become outgoing cylindrical waves at large distances from the disturbance,

2 See for example a discussion by C. S. Yih, Dynamics of Nonhomogeneous Fluids (The Macmillan Company, New York, 1965), p. 69.

${ }^{3}$ G. D. Crapper, J. Fluids Mech. 6, 51 (1959). 
and exist on the downstream side only. In propagating downstream, they radiate a finite amount of energy, and therefore contribute to wave resistance.

\section{FORMULATION OF THE INITIAL VALUE PROBLEM}

We consider the initial value problem of a twodimensional flow of a stratified heavy fluid due to a point source submerged at depth $h$ beneath the initially undisturbed free surface, at $y=0$, of a deep stream having a uniform velocity $U$ in the positive $x$ direction. The strength of the source depends on the time $t$ as a step function $H(t)$. The linearized equations for $y \leq 0$ and $t>0$ in the reference frame fixed at the source are

$$
\begin{gathered}
\rho_{t}+U \rho_{x}+v d \rho_{0} / d y=0, \\
u_{x}+v_{y}=Q H(t) \delta(x) \delta(y+h), \\
\rho_{0}\left(u_{t}+U u_{x}\right)+p_{x}=0, \\
\rho_{0}\left(v_{\imath}+U v_{x}\right)+p_{y}+g \rho=0,
\end{gathered}
$$

where $u$ and $v$ are the $x$ and $y$ components of the perturbation velocity, $\rho$ and $p$ are the perturbation density and pressure measured from the basic flow, $\rho_{0}(y)$ and $p_{0}(y)$, respectively, with $d p_{0} / d y=-g \rho_{0}(y)$. Here the subscripts $x, y, t$ denote partial differentiations. The linearized boundary conditions on the free surface, denoted by $y=\zeta(x, t)$, which is under a constant pressure, are

$$
\begin{array}{ll}
v=\zeta_{t}+U \zeta_{x}, & \text { on } y=0 \\
p=-\zeta p_{0}^{\prime}(y)=-\rho_{0} g \zeta, & \text { on } y=0 .
\end{array}
$$

Furthermore, the perturbations $u, v, \rho, p$ are required to vanish at large distances, as $x^{2}+y^{2} \rightarrow \infty$, $y \leq 0, t>0$. The initial conditions are that $u, v, \rho, p$ and their time derivatives all vanish identically for $t<0$.

We choose an appropriate characteristic length $L$ to form the nondimensional quantities

$$
\begin{aligned}
x_{*} & =x / L, \quad y_{*}=y / L, \quad h_{*}=h / L, \quad t_{*}=U t / L \\
u_{*} & =u / U, \quad v_{*}=v / U, \quad Q_{*}=Q / U L \\
\sigma(y) & =-L \rho_{0}^{\prime}(y) / \rho_{0}(y), \quad \lambda=g L / U^{2} .
\end{aligned}
$$

In what follows the asterisk will be omitted for brevity. Equations (1)-(6) can be combined to yield a single equation for $v$,

$$
\begin{aligned}
& {\left[D^{2} \nabla^{2}-\sigma(y)\left(D^{2} \frac{\partial}{\partial y}-\lambda \frac{\partial^{2}}{\partial x^{2}}\right)\right] v} \\
& \quad=Q D^{2} H(t) \delta(x)\left[\delta^{\prime}(y+h)-\sigma(-h) \delta(y+h)\right]
\end{aligned}
$$

for $y<0$, with the condition

$$
\left(D^{2} \frac{\partial}{\partial y}-\lambda \frac{\partial^{2}}{\partial x^{2}}\right) v=0, \quad(y=0),
$$

where the operator $D \equiv \partial / \partial t+\partial / \partial x$.

We next introduce the Fourier transform with respect to $x$, and the Laplace transform with respect to $t$, defined by

$$
\tilde{\bar{v}}(k, y, s)=\int_{0}^{\infty} e^{-s t} d t \int_{-\infty}^{\infty} e^{-i k x} v(x, y, t) d x
$$

for $k$ real and $\operatorname{Re} s>0$. The tilde and bar over a variable denote, respectively, the Fourier and the Laplace transform. Application of these transforms to (8), (9), under the specified homogeneous initial condition and boundary condition at infinity, yields

$$
\begin{array}{r}
\left(\frac{d^{2}}{d y^{2}}-\sigma \frac{d}{d y}-\left(k^{2}-\lambda_{1} \sigma\right)\right) \tilde{\bar{G}}=\frac{1}{s} \delta(y+h), \\
(y<0), \\
\left(\frac{d}{d y}-\lambda_{1}\right) \tilde{\bar{G}}=0, \quad(y=0),
\end{array}
$$

where

$$
\lambda_{1}=\lambda k^{2} /(k-i s)^{2},
$$

and $G$ is related to $v$ by

$$
v(x, y, t)=Q\left(\frac{\partial}{\partial h}-\sigma(-h)\right) G(x, y, t ; h) .
$$

As we intend to obtain the steady-state solution from the large time limit process, we evaluate, following DePrima and $\mathrm{Wu},{ }^{4}$ the limit of $G(x, y, t)$ by applying the Tauberian theorem which states that

$$
\lim _{t \rightarrow+\infty} G(x, y, t)=\lim _{s \rightarrow 0+} s \bar{G}(x, y, s)
$$

if, and only if

$$
\lim _{t \rightarrow+\infty} \frac{1}{t} \int_{0}^{t} t \frac{\partial G}{\partial t} d t=0 .
$$

Thus, to obtain the large time limit, we only have to evaluate the Laplace transform for small positive s. Only when the necessary and sufficient condition (16) is verified will the asymptotic behavior of $G$ for large $t$ be needed. Following this procedure we determine below the steady-state solution for one of the two cases treated earlier in Ref. 1.

\section{THE PARTICULAR CASE OF CONSTANT o}

For an exponential density distribution

$$
\rho_{0}(y)=\rho_{0}(0) \exp (-y / L), \quad \sigma(y)=1 .
$$

${ }^{4}$ C. R. DePrima and T. Y. Wu, in Proceedings of the IXth International Congress of Applied Mechanics (University of Brussels, Brussels, 1957), Vol. I, p. 388. 


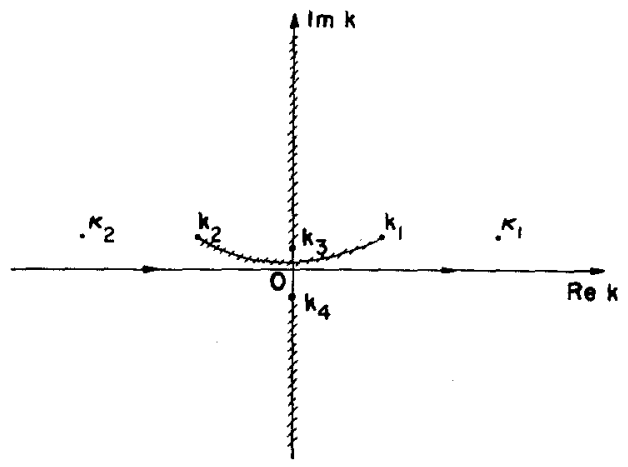

FIG. 1. The branch points and branch cuts in the $k$ plane.

In the above expression, the physical dimension of $y$ is restored to identify the characteristic length $L$. With $\sigma=1$ in (11), the two complementary solutions are $\exp \left(m_{1} y\right)$ and $\exp \left(m_{2} y\right)$, where

$\left.\begin{array}{l}m_{1} \\ m_{2}\end{array}\right\}=\frac{1}{2} \pm M, \quad M=\left(k^{2}-\frac{\lambda k^{2}}{(k-i s)^{2}}+\frac{1}{4}\right)^{\frac{1}{2}}$.

Clearly, $M$ can be factorized as

$M=\left[\left(k-k_{1}\right)\left(k-k_{2}\right)\left(k-k_{3}\right)\left(k-k_{4}\right)\right]^{\frac{1}{2}}(k-i s)^{-1}$,

showing that $M$ has in the complex $k$ plane, four branch points at $k_{1}, k_{2}, k_{3}, k_{4}$. For small positive $s$, we find that

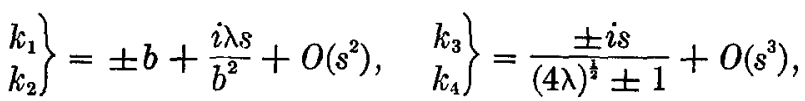

$$
b=\left(\lambda-\frac{1}{4}\right)^{\frac{1}{2}} .
$$

Hence for $\lambda>\frac{1}{4}, k_{1}$ is located in the first quadrant, $k_{2}$ in the second quadrant of the $k$ plane, $k_{3}$ on the positive, and $k_{4}$ on the negative imaginary $k$ axis. In practical interest $\lambda$ is generally large. In order to ensure the convergence of the Fourier inversion integral, we require that $M \rightarrow \pm k$ as $k \rightarrow \pm \infty$ along the real axis. This branch of $M$ can be achieved by introducing a branch cut between $k_{1}$ and $k_{2}$ in

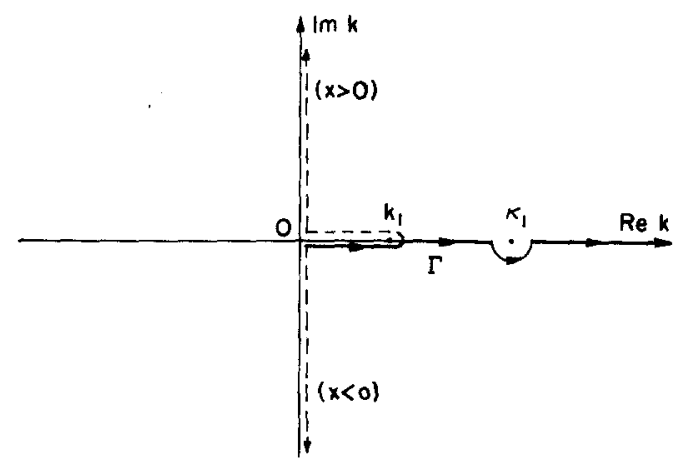

FIG. 2. The path of integration $\Gamma$. the upper-half $k$ plane, and a cut from $+i \infty$ to $k_{3}$, and another from $k_{4}$ to $-i \infty$ along the imaginary $k$ axis, as shown in Fig. 1. For small positive $s$, the function $M(k, s)$ is then analytic in the entire cut $k$ plane, which includes in particular the entire real $k$ axis. In accordance with this branch of $M$, the solution of (11) satisfying condition (12) and the required jump across $y=-h$, and vanishing at $y=-\infty$, is

$\tilde{\bar{G}}=-\frac{\exp [(y+h) / 2]}{2 s M}$

$$
\begin{gathered}
\left(\exp (-|y+h| M)-\frac{m_{2}-\lambda_{1}}{m_{1}-\lambda_{1}} e^{(y-h) M}\right), \\
(y<0) .
\end{gathered}
$$

Aside from the four branch points of $M, \widetilde{\bar{G}}$ has poles at the zeros of $\left(m_{1}-\lambda_{1}\right)$. For $\lambda>\frac{1}{2}, \tilde{\bar{G}}$ has only two simples poles at $k=\kappa_{1}, \kappa_{2}$, where

$\kappa_{1}=+\lambda+2 i s+O\left(s^{2}\right), \quad \kappa_{2}=-\lambda+2 i s+O\left(s^{2}\right)$.

For $\operatorname{Re} s>0, \kappa_{1}$ and $\kappa_{2}$ are located in the upper-half $k$ plane, symmetrical about the imaginary axis (see Fig. 1).

Now, by applying the Fourier inversion theorem,

$$
\begin{aligned}
\bar{G}(x, y, s) & =\frac{1}{2 \pi} \int_{-\infty}^{\infty} e^{i k x} \tilde{\bar{G}}(k, y, s) d k \\
& =\frac{1}{\pi} \operatorname{Re} \int_{0}^{\infty} e^{i k x} \tilde{\bar{G}}(k, y, s) d k
\end{aligned}
$$

in which the last expression follows from the symmetry of the branch cuts and the location of the poles of $\tilde{\bar{G}}$ with respect to the imaginary axis and that $\tilde{\bar{G}}(-k, y, s)$ is complex conjugate to $\tilde{\widetilde{G}}(k, y, s)$, with $s$ real, positive. We next seek the limit of $\bar{G}$ as $s \rightarrow 0+$. In this limit, $k_{1} \rightarrow b, k_{2} \rightarrow-b, k_{3} \rightarrow 0$, $\kappa_{1} \rightarrow \lambda, \kappa_{2} \rightarrow-\lambda$, all from above the real $k$ axis, and $k_{4} \rightarrow 0$ from below. Consequently, we may deform the path of integration in (22) to $\Gamma$ which lies along the positive real $k$ axis except that it circumvents below the branch cut from $k=0$ to $b$, and also below the pole at $k=\lambda$, as shown in Fig. 2. Hence, by the Tauberian theorem (15), the steady-state solution of $G$ is

$$
\begin{aligned}
G(x, y) & =-\frac{1}{2 \pi} e^{(y+h) / 2} \operatorname{Re} \int_{0 \Gamma}^{\infty} e^{i k x}(\exp (-|y+h| \\
& \left.-\frac{\left(\lambda-\frac{1}{2}\right)+B}{\left(\lambda-\frac{1}{2}\right)-B} \exp [(y-h) B]\right) \frac{d k}{B},
\end{aligned}
$$

where

$$
B=\left(k^{2}-b^{2}\right)^{\frac{1}{2}}, \quad b=\left(\lambda-\frac{1}{4}\right)^{\frac{1}{2}},
$$


provided the necessary and sufficient condition (15) is satisfied. The fulfilment of this condition can be shown in a manner similar to that by De Prima and Wu. ${ }^{4}$ In short, it follows from the asymptotic behavior that the time-dependent part of $G$ falls off like $t^{-\alpha} \exp (i \omega t)$ for $t$ large, with $0<\alpha<1$ and $\omega$ real, nonzero. The details of this verification, however, will be left out here.

The resulting branch cuts for the integrand in (23) are different from those arbitrarily chosen in Ref. 1. Without the guidance of the corresponding initial-value problem, the branch cuts along the imaginary $k$ axis disappear completely, and the branch cuts from $k= \pm b$ on the different sides of the real $k$ axis lead to erroneous result of the internal waves, as will be shown below.

The contour $\Gamma$ may be further deformed for $x<0$ to a new path along the entire negative imaginary $k$ axis, from $k=0$ to $-i \infty$ (see Fig. 2). For $x>0$, we may introduce a closed contour by adding to $\Gamma$ a large circular are $|k|=K$ from $k=K$ to $i K$, returning to $k=+i 0$ along the positive imaginary axis, and then circumventing the branch cut around the point $k=b$ back to $k=-i 0$. Within this contour there is only one simple pole at $k=\lambda$. By applying the theorem of residue, we obtain, after some straightforward manipulation, the following result, valid for $\lambda>\frac{1}{2}$ :

$$
\begin{gathered}
G(x, y)=G_{1}(x, y)+G_{2}(x, y)+G_{3}(x, y) \\
G_{1}=[2-(1 / \lambda)] H(x) e^{(1-\lambda) h+\lambda y} \sin \lambda x \\
G_{2}=\frac{H(x)}{2 \pi} e^{\frac{1}{2}(y+h)} \operatorname{Re} \int_{-\pi / 2}^{\pi / 2} \frac{1}{i} e^{i X \cos \beta} \\
\left\{e^{-i Y_{1 \sin \beta}}-\frac{\lambda-\frac{1}{2}+i b \sin \beta}{\lambda-\frac{1}{2}-i b \sin \beta} e^{-i Y_{u s i n} \beta}\right\} d \beta, \\
G_{3}=-\frac{1}{2 \pi} e^{\frac{1}{2}(y+h)} \operatorname{Re} \int_{0}^{\infty} e^{-X \sin b t} \\
\cdot\left\{e^{-i Y_{i} \cos h t}-\frac{\lambda-\frac{1}{2}+i b \cosh t}{\lambda-\frac{1}{2}-i b \cosh t} e^{-i Y_{u \cos b t}}\right\} d t, \\
X=b|x|, \quad Y_{l}=b|y+h|, \\
Y_{u}=b(h-y), \quad b=\left(\lambda-\frac{1}{4}\right)^{\frac{1}{2}},
\end{gathered}
$$

where $H(x)=1$ or 0 according as $x>$ or $<0$. In the above expression for $G, G_{1}$ is the contribution from the residue, $G_{2}$ comes from the integration around the right half of the branch cut, and $G_{3}$ from the integration along the imaginary axis. Since $G_{1}$ satisfies the Laplace equation, it represents an irrotational surface wave. As will be seen below, $G_{2}$ represents the internal gravity waves arising from the density stratification, and $G_{3}$ exhibits a local effect since it decays more rapidly than $G_{2}$ at large distances.

\subsection{Asymptotic Behavior of the Flow Field; Internal Waves}

The asymptotic behavior of $G_{2}$ and $G_{3}$ at large distances can be evaluated by usual asymptotic methods. $G_{2}$ contains integrals of the form

$$
\begin{gathered}
F_{2}(X, Y)=\int_{-\pi / 2}^{\pi / 2} e^{f(\beta)} \varphi(\sin \beta) d \beta, \\
f(\beta)=i X \cos \beta-i Y \sin \beta=i R \cos (\beta+\theta), \\
R^{2}=X^{2}+Y^{2}, \quad \theta=\tan ^{-1}(Y / X),
\end{gathered}
$$

where $X$ and $Y$ are real positive parameters. For $R$ large, $F_{2}$ can be evaluated by the method of steepest descent. By treating $\beta$ as a complex variable, $\beta=\xi+i \eta$, the integrand has a saddle point at $\beta_{0}=$ $-\theta$, which is located in the region $-\frac{1}{2} \pi<\beta_{0}<0$. The original path of integration along the real $\beta$-axis, which is a level line, can be deformed to the following steepest paths: (i) path $C_{1}$ from $\beta_{1}=-\frac{1}{2} \pi$ to $\beta_{2}=$ $-(\pi / 2+\theta)+i \infty$ along $\cosh \eta=\sin \theta \sec (\xi+\theta)$, then (ii) path $C_{2}$ from $\beta_{2}$ to $\beta_{3}=\left(\frac{1}{2} \pi-\theta\right)-i \infty$ along cosh $\eta=\sec (\xi+\theta)$, and finally (iii) path $C_{3}$ from $\beta_{3}$ to $\beta_{4}=\frac{1}{2} \pi$ along $\cosh \eta=-\sin \theta$ sec $(\xi+\theta)$. The most significant contributions come from the integration along three short stretches of these paths near $\beta=-\frac{1}{2} \pi,-\theta$, and $\frac{1}{2} \pi$. If in the integrals along $C_{1}$ and $C_{3}$ one further expands

$$
\begin{aligned}
& \left(\sinh ^{2} \eta+\cos ^{2} \theta\right)^{1} \\
& \quad \sim \cos \theta+\frac{1}{2} \sec \theta \sinh ^{2} \eta \\
& \sim \sinh \eta+\frac{1}{2} \cos ^{2} \theta \cosh \eta
\end{aligned}
$$

$(\sinh \eta<\cos \theta)$, $(\sinh \eta>\cos \theta)$,

one finally obtains, noting that $\varphi(+1)$ equals the complex conjugate of $\varphi(-1)$ in the present case, the following result:

$$
\begin{aligned}
& F_{2}(X, Y) \sim(2 \pi / R)^{\frac{1}{3}} e^{i(R-\pi / 4)} \varphi(-\sin \theta) \\
& \quad+2 i \operatorname{Re}\left\{\varphi ( 1 ) e ^ { - i Y } \left[X^{-1}\left(1-e^{-R \cos ^{2} \theta}\right)\right.\right. \\
& \left.\left.\quad+(\pi / 4 R)^{\frac{1}{2}}(1-i \sin \theta) \operatorname{erfc}\left(R^{\frac{3}{2}} \cos \theta\right)\right]\right\}+O\left(R^{-\frac{1}{2}}\right) .
\end{aligned}
$$

This asymptotic representation of $F_{2}$ is valid uniformly for $R \gg 1,0 \leq \theta \leq \frac{1}{2} \pi$. In particular, we have, respectively, for (i) $X \gg 1, Y$ arbitrary; and (ii) $X \ll 1 \ll Y$

$$
\begin{array}{r}
F_{2}(X, Y) \sim(2 \pi / R)^{\frac{1}{i(R-x / 4)} \varphi(-\sin \theta)} \\
+(2 i / X)\left\{\operatorname{Re}\left[\varphi(1) e^{-i Y}\right]\right\}+O\left(R^{-\frac{1}{3}}\right) \\
F_{2}(X, Y) \sim(2 \pi / Y)^{\frac{1}{2}}\left\{\operatorname{Re}\left[\varphi(1) e^{-i(Y-x / 4\rangle}\right]\right\} \\
+O(X / Y)
\end{array}
$$


In ( $24 \mathrm{~d}), G_{3}$ contains integrals of the form

$$
\begin{aligned}
F_{3}(X, Y) & =\int_{0}^{\infty} e^{-X \sinh t-i Y_{\cosh t}} \varphi(\cosh t) d t \\
& =\int_{0}^{\infty} e^{-i \sinh (t+i \theta)} \varphi(\cosh t) d t .
\end{aligned}
$$

For $R$ large, one may regard $t$ to be complex, $t=$ $\xi+i \eta$, and deform the path of integration to the steepest path from $t=0$ and $t=\infty-i \theta$ along $\cosh \xi=\sin \theta \csc (\eta+\theta)$. The resulting integral is similar to that along $C_{3}$ for $F_{2}$. We finally obtain

$$
\begin{aligned}
F_{3}(X, Y) & \sim \varphi(1) e^{-i \mathrm{Y}}\left[X^{-1}\left(1-e^{-R_{\cos ^{2} \theta}}\right)\right. \\
& \left.+(\pi / 4 R)^{\frac{1}{2}}(1-i \sin \theta) \operatorname{erfc}\left(R^{\frac{1}{3}} \cos \theta\right)\right] \\
& +O\left(R^{-\frac{3}{3}}\right) .
\end{aligned}
$$

Hence

$$
F_{3}(X, Y) \sim e^{-i Y}[\varphi(1) / X]+O\left(X^{-2}\right), \quad(X \gg 1),
$$

$$
F_{3}(X, Y) \sim \varphi(1)(\pi / 2 Y)^{\frac{1}{2}} e^{-i(Y+x / \phi)}+O(X / Y),
$$

$$
(X \ll 1 \ll Y) \text {. }
$$

Making use of the above asymptotic expansions in (24), we obtain for $X \gg 1$,

$$
\begin{aligned}
& G_{2}(x, y) \sim H(x) e^{\frac{1}{2}(y+h)} \\
& \cdot\left(\frac{\sin \left(R_{l}-\frac{1}{4} \pi\right)}{\left(2 \pi R_{l}\right)^{\frac{1}{2}}}-\frac{\sin \left(R_{u}-\frac{1}{4} \pi\right)}{\left(2 \pi R_{u}\right)^{\frac{3}{3}}}+\frac{2 b \sin \theta_{u}}{\left(2 \pi R_{u}\right)^{\frac{3}{2}}}\right. \\
& \left.\frac{\left(\lambda-\frac{1}{2}\right) \cos \left(R_{u}-\frac{1}{4} \pi\right)+b \sin \theta_{u} \sin \left(R_{u}-\frac{1}{4} \pi\right)}{\left(\lambda-\frac{1}{2}\right)^{2}+b^{2} \sin ^{2} \theta_{u}}\right) \\
& -2 H(x) G_{3}(x, y)+O\left(R^{-\frac{3}{2}}\right), \\
& G_{3}(x, y) \sim-(1 / 2 \pi) e^{\frac{1}{2}(y+h)}\left\{X^{-1}\left(\cos Y_{l}-\cos Y_{u}\right)\right. \\
& \left.\quad+\left(2 b / \lambda^{2} X\right)\left[b \cos Y_{u}-\left(\lambda-\frac{1}{2}\right) \sin Y_{u}\right]\right\} \\
& \quad+O\left(X^{-2}\right),
\end{aligned}
$$

where

$$
\begin{aligned}
& X=b|x|, \\
& Y_{\imath}=b|y+h|=R_{\imath} \sin \theta_{l}, \\
& Y_{u}=b(h-y)=R_{u} \sin \theta_{u} .
\end{aligned}
$$

For $X \ll 1 \ll Y$, we have

$$
\begin{aligned}
G_{2} & +G_{3} \\
\sim & -\frac{1}{2} e^{\frac{1}{2}(u+h)}\left(\frac{\sin \left(Y_{l}-\frac{1}{4} \pi\right)}{\left(2 \pi Y_{l}\right)^{\frac{1}{2}}}-\frac{\sin \left(Y_{u}-\frac{1}{4} \pi\right)}{\left(2 \pi Y_{u}\right)^{\frac{1}{2}}}\right. \\
& \left.-\frac{2 b}{\lambda^{2}} \frac{\left(\lambda-\frac{1}{2}\right) \cos \left(Y_{z}-\frac{1}{4} \pi\right)+b \sin \left(Y_{u}-\frac{1}{4} \pi\right)}{\left(2 \pi Y_{u}\right)^{\frac{1}{2}}}\right) \\
& +O(X / Y) .
\end{aligned}
$$

This result holds valid for both $x$ positive and negative.

Summarizing the above result, we note that $G_{1}$ represents an irrotational surface wave which decays exponentially into the interior and propagates to $x=+\infty$ without attenuation. Its effect on wave drag has been studied in detail ${ }^{1} . G_{2}$ represents the internal waves produced in the nonhomogeneous medium. At large distances, the leading term of $G_{2}$, given by the first term in (29a), exhibits two cylindrical waves centered, respectively, at $(0,-h)$ and $(0, h)$, which exist only on the downstream side, $x>0$, and are attenuated like $R^{-\frac{3}{2}}$ (when the three terms are considered together). This wave mode was calculated incorrectly in Ref. (1) because of the wrong branch cuts chosen, yielding the wrong result that the internal waves exist on both the downstream and upstream side. Since the amplitude of these internal waves decays like $R^{-1}$, their energy falls off like $R^{-1}$ as $R \rightarrow \infty$, and hence the rate of transfer of the wave energy across a. large circle of radius $R$ will be constant. Therefore, on physical grounds, we conclude that this wave mode, like the surface wave, also radiates energy, and consequently contributes a finite wave resistance. The next order term of $G_{2}$ is equal to $-2 G_{3}$ for $x$ positive and large. This term and $G_{3}$ combined produce a secondary internal wave mode which oscillates only in the vertical direction, exists on both sides of $x=0$ (actually an odd function of $x$ ), and decays like $|x|^{-1}$ for $|x|$ large. The higher-order terms of $G_{2}$ and $G_{3}$ can be seen to be less significant.

In a small neighborhood of the singularity, $X$ and $Y_{l}$ being both small, it can be shown, by expanding the integrand of $G_{2}$ in a Taylor series and integrating termwise, that $G_{2}$ is bounded as $R_{l} \rightarrow 0$. For the integral representation of $G_{3}$, one may use the formula for the Bessel function of the second kind,

$$
Y_{0}(z)=\frac{1}{\pi} \int_{0}^{\pi} \sin (z \sin \beta) d \beta-\frac{2}{\pi} \int_{0}^{\infty} e^{-z \sinh t} d t,
$$

and show that

$$
\begin{aligned}
G_{3}(X, Y) & \sim \frac{1}{4} Y_{0}\left(R_{l}\right)+O(1) \\
& \sim(1 / 2 \pi) \log R_{l}+O(1) \text { as } R_{t} \rightarrow 0 .
\end{aligned}
$$

This result implies that the inertial effect predominates in the neighborhood of $R_{2}=0$, and hence $G$ retains there its behavior in a homogeneous medium, as should be expected.

The case of arbitrary $\sigma(y)$, but with $\lambda$ large, will be discussed later in a separate work.

\section{ACKNOWLEDGMENT}

This work has been supported in part by the Office of Naval Research under Contract Nonr 220(35). 